\title{
Retinopatía del prematuro
}

\author{
Retinopathy of prematurity
}

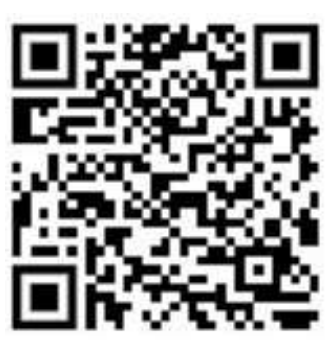

${ }^{1}$ Médico general, graduado de la Universidad de Ciencias Médicas (UCIMED), médico en Hospital La California, San José, Costa Rica. Código médico: 15305 fe co 07@hotmail.com

${ }^{2}$ Médico general, graduada de la Universidad de Ciencias Médicas (UCIMED), médico en Arvesalud consultorios limitada y Consultorio Médico El Llano, San José, Costa Rica. Código médico: 15245 stef1794@hotmail.com

${ }^{3}$ Médico general, graduada de la Universidad de Ciencias Médicas (UCIMED) médico investigadora independiente, Heredia, Costa Rica. Código médico: 15243

danielad0593@gmail.com

${ }^{4}$ Médico general, graduada de la Universidad de Ciencias Médicas (UCIMED), médico en Hospital La California, San José, Costa Rica. Código médico: 15340 jazmin-qc@hotmail.com
'Dr. Federico Cortés Bejarano

Hospital La California, San José, Costa Rica

fe co 07@hotmail.com

https://orcid.org/0000-0001-8116-0900

${ }^{2}$ Dra. Estefany Isabel Cortés Morales

Arvesalud consultorios limitada y Consultorio Médico El Llano, San José, Costa Rica stef1794@hotmail.com

https://orcid.org/0000-0002-6304-0240

${ }^{3}$ Dra. Daniela Duarte Núñez Investigadora independiente, Heredia, Costa Rica

fe co 07@hotmail.com

https://orcid.org/0000-0001-6329-9669

${ }^{4}$ Dra. Jazmín Victoria Quesada Campos Hospital La California, San José, Costa Rica jazmin-qc@hotmail.com https://orcid.org/0000-0002-4211-6437

$\begin{array}{ccc}\text { RECIBIDO } & \text { CORREGIDO } & \text { ACEPTADO } \\ 28 / 1 / 2019 & 8 / 2 / 2019 & 24 / 2 / 2019\end{array}$

\section{RESUMEN}

La retinopatía del prematuro sigue siendo la principal causa de ceguera infantil que afecta principalmente a los bebés prematuros y de bajo peso al nacer. La prevención del parto prematuro es la estrategia más efectiva para reducir la incidencia de retinopatía del prematuro. La identificación y el tratamiento oportunos son esenciales para prevenir la pérdida innecesaria de la visión causada por retinopatía del prematuro. Se ha asociado con múltiples factores de riesgo entre los principales el bajo peso al nacer, edad gestacional y utilización de oxígeno suplementario, entre otros. Su cuadro clínico se caracteriza por una membrana retrolental que origina leucocoria y eventualmente sin tratamiento con complicaciones potenciales como glaucoma, catarata, miopía y ceguera, siendo esta última la más grave. El estándar de tratamiento actual sigue siendo la terapia ablativa a través de la fotocoagulación con láser de la retina isquémica avascular periférica y actualmente se están realizando estudios con anti VEGF los cuales han demostrado beneficios significativos, sin embargo aún no han sido aprobado por la FDA para su utilización clínica. 
PALABRAS CLAVES: retinopatía del prematuro; recién nacido prematuro; recién nacido de bajo peso; ceguera; agudeza visual.

\section{ABSTRACT}

Retinopathy of prematurity continues to be the leading cause of childhood blindness that primarily affects premature and low birth weight babies. The prevention of preterm birth is the most effective strategy to reduce the incidence of retinopathy of prematurity. Timely identification and treatment are essential to prevent the unnecessary loss of vision caused by retinopathy of prematurity. It has been associated with multiple risk factors among the main low birth weight, gestational age and use of supplemental oxygen, among others. Its clinical picture is characterized by a retrolental membrane that causes leukocoria and eventually without treatment with potential complications such as glaucoma, cataract, myopia and blindness, the latter being the most serious. The current standard of treatment continues to be ablative therapy through laser photocoagulation of the peripheral avascular ischemic retina and studies are currently being conducted with anti VEGF which have shown significant benefits, however, they have not yet been approved by the FDA for its clinical use.

KEYWORDS: retinopathy of prematurity; premature infant; low birthweight infant; blindness; visual acuity.

\section{INTRODUCCIÓN}

La retinopatía del prematuro (ROP) es un desorden retiniano vasoproliferativo único para infantes prematuros $(1,2)$. Esta es una compleja enfermedad de la vasculatura retiniana inmadura en neonatos pretérminos (3). La ROP es una vitreoretinopatía proliferativa periférica (4).

No se conocen completamente los factores de riesgo pero los más importantes son ser recién nacido pretérmino e inmadurez retiniana al nacer (3). La mayoría de los casos de la retinopatía del prematuro ocurren en pacientes extremadamente prematuros (menores de 28 semanas al nacer) y también en infantes con bajo peso al nacer $(5,6)$. Actualmente es la causa principal prevenible de ceguera infantil en Latinoamérica (7).

Los avances en la medicina neonatal han permitido el aumento en la supervivencia en los infantes extremadamente prematuros, en parte debido al uso de oxígeno suplementario en incubadoras cerradas, a la vez aumentando la incidencia del ROP $(6,8)$. La evolución del ROP puede ir desde regresión espontánea hasta formas más graves con pérdida de agudeza visual y ceguera $(4,5)$. 


\begin{tabular}{|c|c|c|c|c|c|c|c|}
\hline \multirow{3}{*}{$\begin{array}{l}\text { Años a } \\
\text { valorar }\end{array}$} & \multicolumn{7}{|c|}{ Factores de riesgo } \\
\hline & \multirow{2}{*}{ Prematuridad } & \multicolumn{3}{|c|}{ Bajo peso al nacer } & \multirow{2}{*}{$\begin{array}{l}\text { Oxigenoterapia } \\
\text { altas dosis }\end{array}$} & \multirow{2}{*}{$\begin{array}{c}\text { Otras } \\
\text { patologias }\end{array}$} & \multirow{2}{*}{$\begin{array}{l}\text { Cuidados } \\
\text { neonatales }\end{array}$} \\
\hline & & & $<1000 \mathrm{~g}$ & $1000-1500 \mathrm{~g}$ & & & \\
\hline 1940-1950s & + & + & $\begin{array}{l}\text { Mortalidad alta } \\
\text { Sin ROP }\end{array}$ & $\begin{array}{c}\text { Sobrevivian } \\
\text { ROP +++ }\end{array}$ & ++++ & + & Pobres \\
\hline 1960-1970s & ++ & ++ & $\begin{array}{c}\text { Mortalidad } \\
\text { moderada ROP+ }\end{array}$ & $\begin{array}{c}\text { Sobrevivian } \\
\text { ROP ++ }\end{array}$ & ++ & + & Moderados \\
\hline 1980-2000s & ++++ & ++++ & $\begin{array}{c}\text { Mortalidad baja } \\
\text { ROP+++ }\end{array}$ & $\begin{array}{c}\text { Mortalidad muy } \\
\text { baja Sin ROP }\end{array}$ & + & $+/-$ & Excelentes \\
\hline
\end{tabular}

Fuente: Gilbert C. Retinopathy of prematurity: A global perspective of the epidemics, population of babies at risk and implications for control. Early Human Development.

TABLA 2. ROP como causa de ceguera a nivel mundial, según la región geográfica, niños examinados, problema de salud pública.

\begin{tabular}{|c|c|c|c|}
\hline \multirow[b]{2}{*}{ Región geográfica } & \multicolumn{2}{|c|}{ Niños examinados } & \multirow{2}{*}{$\begin{array}{l}\text { Problema de salud pública según } \\
\text { tasas de mortalidad }\end{array}$} \\
\hline & $\begin{array}{c}\text { Número }>50000 \text { niños } \\
\text { examinados }\end{array}$ & $\begin{array}{l}\text { Porcentaje (\%) } \\
\quad>15000\end{array}$ & \\
\hline $\begin{array}{c}\text { Antiguas Economías } \\
\text { Socialistas }\end{array}$ & 15000 & 37.4 & \multirow{2}{*}{$\begin{array}{l}\text { Alto riesgo de ceguera por ROP. } \\
\text { Cuidados neonatales y tamizaje no } \\
\text { siempre adecuado }\end{array}$} \\
\hline $\begin{array}{l}\text { Latinoamérica y el } \\
\text { Caribe }\end{array}$ & 24000 & 23.4 & \\
\hline $\begin{array}{l}\text { Economías } \\
\text { Comerciales } \\
\text { Establecidas }\end{array}$ & 4500 & 10 & $\begin{array}{l}\text { Bajo riesgo de ceguera por ROP. } \\
\text { Cuidados neonatales y tamizaje } \\
\text { adecuados }\end{array}$ \\
\hline China & 4000 & 1.9 & \multirow{4}{*}{$\begin{array}{l}\text { Alto riesgo de ceguera por ROP. } \\
\text { Cuidados neonatales y tamizaje no } \\
\text { siempre adecuado }\end{array}$} \\
\hline $\begin{array}{c}\text { Otros de Asia y las } \\
\text { Islas }\end{array}$ & 3500 & 1.8 & \\
\hline India & 500 & 0.1 & \\
\hline $\begin{array}{l}\text { Medio Oriente } \\
\text { creciente }\end{array}$ & 300 & 0.1 & \\
\hline África subsahariana & 200 & 0 & $\begin{array}{l}\text { Bajo riesgo de ceguera por ROP. } \\
\text { Cuidados neonatales no bien } \\
\text { desarrollados }\end{array}$ \\
\hline \multicolumn{4}{|c|}{$\begin{array}{l}\text { Nota: La información de África Subsahariana muestra que la retinopatía del prematuro afecta solamente niños } \\
\text { caucásicos y asiáticos. Como ningún otro país de la región tuvo niños ciegos por ROP, la proporción de la región se ha } \\
\text { indicado con } 0 \% \text {. } \\
\text { Fuente: Gilbert C. Retinopathy of prematurity: A global perspective of the epidemics, population of babies at risk and } \\
\text { implications for control. Early Human Development. }\end{array}$} \\
\hline
\end{tabular}

\section{HISTORIA}

La ROP fue descrita por primera vez en USA en 1940 inicialmente como Fibroplasia retrolental por Clifford Stewart, posteriormente Theodore Terry le dio seguimiento al estudio y la describió como Retinopatía del prematuro en 1946, asociándose tiempo después al uso excesivo del oxígeno $(9,10)$. Se describen 3 epidemias a lo largo de la historia que datan la primera en 1950 asociada a los altos complementos de oxígeno no 
monitorizado. La segunda epidemia ocurrió en 1970 en países desarrollado como consecuencia de la alta tasa de supervivencia en bebés extremadamente prematuros. La tercera epidemia se observó en países en vías de desarrollo entre el año 2000 y 2010 como consecuencia de los avances en la reducción de la mortalidad en bebés extremadamente prematuros $(11,12)$. Históricamente en Costa Rica en 1981 se implementaron políticas gubernamentales nacionales que incluyen prevención, detección, tratamiento y tamizaje para ROP financiadas por el estado, a diferencia de otros países de Latinoamérica que adoptaron este programa más tardíamente en el año 2012 (7). Ver TABLA 1.

\section{EPIDEMIOLOGIA}

Mundialmente cerca del $10 \%$ de los nacimientos son pretérminos, aproximadamente antes de la semana 37 de gestación. En infantes con edad gestacional menor de 27 semanas se reporta ROP (cualquier estadio) en un $73 \%$ y Retinopatía severa del prematuro se reporta en un $35 \%$ (6). Actualmente es la tercera causa de ceguera infantil en Estados Unidos, hasta un 14\% (13). En un estudio de Early Treatment for Retinopathy of Premature (ETROP) se reportó una incidencia de ROP de hasta un $65.8 \%$ en infantes con peso al nacer $<1251$ gramos (14). En el 2010, se estimó que a nivel mundial 184.700 bebés prematuros desarrollarían ROP, y de ellos, 20.000 quedarían ciegos o gravemente discapacitados visualmente (13). En total aproximadamente el $90 \%$ de los infantes con factores de riesgo para ROP terminan teniendo una regresión y resolución espontánea, y menos del $10 \%$ progresan a una enfermedad grave (3). Ver TABLA 2.

\section{PATOGENIA}

La angiogenia retiniana inicia durante la semana 16 de gestación desde el tejido mesenquimal, creciendo de forma centrífuga desde el disco óptico hasta alcanzar la ora serrata nasal en torno a las 36 semanas y la ora serrata temporal en torno a las 40 semanas. La ROP resulta por crecimiento anormal de estos vasos retinianos en un infante prematuro debido a una compleja interacción entre el factor de crecimiento endotelial vascular (VEGF) y el factor de crecimiento similar a la insulina (IGF-I) $(1,3)$. Se han identificado 2 factores angiogénicos que participan en la vascularización de la retina, el VEGF y el IGF-I. EI VEGF es secretado por los astrocitos de la retina avascular y se produce en respuesta a la hipoxia; el IGF-I es principalmente de aporte exógeno (placenta y líquido amniótico) pero, a diferencia del anterior, es un factor independiente del oxígeno y es permisivo de la acción del VEGF. Si los niveles de IGF-I están disminuidos no se lleva a cabo la vascularización normal de la retina, a pesar de VEGF $(4,15)$. La patogenia consta de 2 fases:

Fase I (vasobliterante): caracterizada por un proceso vasobliterativo relacionado con la exposición de la retina inmadura del ojo en desarrollo en recién nacidos extremadamente prematuros a niveles normales o más altos de tensión de oxígeno en comparación con los valores fetales. Mientras que la saturación de oxígeno fetal oscila entre $50 \%-70 \%$, la saturación de oxígeno inmediatamente después del nacimiento aumenta a un $80 \%$ - $100 \%$. Este aumento de 
saturación de oxígeno da como resultado una marcada disminución en la confluencia del VEGF que conduce a la hipoxia de la zona vascular de la retina. La hiperoxia asociada con oxígeno suplementario aumenta esta regulación a la baja de la angiogénesis. Este proceso comienza al nacer y continúa hasta alrededor de las 32 semanas según la edad gestacional por fecha de última regla (11).

Fase II (vasoproliferativa): esta fase, que comienza alrededor de las 36 semanas según la edad gestacional por fecha de última regla, está marcada por la sobreproducción de VEGF y Eritropoyetina (EPO) causada por una retina previamente hipóxica, conduciendo a la vasoproliferación. Los factores de crecimiento independientes del oxígeno como el IGF-I también juegan un papel vital durante la fase 2 , aumentando las concentraciones lo suficiente hasta activar la vía del VEGF. Si la vasoproliferación anormal progresa a través de la retina hacia el vítreo, sangre y fuga de líquidos se extenderá a las diferentes partes del ojo, llevando a la formación de cicatriz y tracción retiniana que a su vez conduce a desprendimiento de la retina completo y en última instancia ceguera permanente $(6,15)$.

\section{FACTORES DE RIESGO}

Los factores más importantes para el desarrollo de la retinopatía son la prematuridad y el bajo peso al nacimiento y sobre ellos actuando otros factores, entre los cuales el oxígeno parece ser el más importante pero no imprescindible $(1,4)$.

- Peso al nacer: los autores proponen que en los países industrializados clasifican como en alto riesgo neonatos con peso $<1500 \mathrm{~g}$ y en países en vías de desarrollo el corte es de $<2000 \mathrm{~g}$ (7).

- Edad gestacional: a menor edad gestacional existe incremento en incidencia de ROP. En un estudio en el McMaster Children's Hospital se determinó la incidencia de ROP en infantes de distintas semanas de gestación. El estudio concluyó una incidencia del $96 \%$ en ROP en infantes con edad gestacional $<24$ semanas, un $83.4 \%$ de incidencia en ROP en infantes con edad gestacional de 25-26 semanas, una incidencia del $61.9 \%$ en ROP en infantes con edad gestacional de 27-28 semanas, un $43.6 \%$ de incidencia en infantes con edad gestacional de 29-30 semanas y una incidencia $34.1 \%$ en ROP en infantes con edad gestacional de 31 semanas o más (16).

- Oxígeno: diferentes estudios han probado que las fluctuaciones de oxígeno está reconocido como un factor de riesgo para ROP. Fluctuaciones en oxígeno en diferentes tiempos hasta 30 días después del nacimiento aumentan las probabilidades de que un infante pretérmino desarrolle ROP severa. Estas fluctuaciones en el oxígeno actuando en la retina del infante pretérmino pueden ser por apena, bradicardia, cambios en la hemoglobina fetal - adulta, cortocircuitos de sangre en pulmones, cambios en el $\mathrm{CO} 2 \mathrm{y}$ temperatura. "The Neonatal Research Network"comparó rangos de saturación de oxigeno desde $85 \%-89 \%$ versus $91 \%-95 \%$ entre 1316 infantes nacidos entre $24-28$ 
semanas de edad gestacional. Retinopatía severa ocurrió en menor frecuencia en sobrevivientes del grupo de saturación de $85 \%$ $89 \%(17,18)$. Se ha visto que una saturación de oxígeno >93\% aumenta el riesgo de retinopatía del prematuro severa, con necesidad de tratamiento (18).

- Genética: estudios demuestran que $70 \%-80 \%$ de la susceptibilidad de ROP está condicionada por factores genéticos. La heredabilidad de ROP está estimada en un $72.8 \%$. El desarrollo neurovascular de la retina está genéticamente condicionado y está modificado por un número grande de genes y citoquinas que oscilan dependiendo de las condiciones locales y generales del ambiente (19).

- Anemia ferropénica materna: se observó un incremento marcado en el desarrollo de ROP en infantes prematuros nacidos de madres con ferropenia al compararse con infantes nacidos de madres con niveles férricos séricos normales (1).

Otros factores de riesgo también asociados a ROP como sepsis, hiperglicemia, anemia, hemorragia intraventricular, hemorragia fetal, ductus persistente, síndrome de distress respiratorio, apnea, displasia broncopulmonar, transfusiones sanguíneas frecuentes, ventilación mecánica prolongada, exposición a la luz, concentraciones bajas de cortisol, raza blanca, hipertiroidismo materno, nacimiento extra hospitalario, bajos niveles de IGF-I, tratamiento con insulina, tratamiento con corticoesteroides y nutrición parenteral prolongada $(4,6,13,20)$.

\section{FACTORES PROTECTORES}

- Leche materna: el amamantamiento con lactancia materna actúa como un factor protector en cualquier estadio de ROP. Debido a que la leche materna contiene vitamina $\mathrm{C}, \mathrm{E}$, betacarotenos y propiedades antioxidantes mayores que la fórmula. Así mismo sustancias inmunomoduladoras como $\lg A$ secretora, lactoferrina, lisozima y citoquinas. Estos factores se piensan que influencian la inmunidad del infante, lo que puede explicar la menor incidencia de ROP y riesgo de enterocolitis necrotizante (21).

Otros factores protectores como hipoxia, enterocolitis necrotizante, enfermedad hemolítica, nutrición rica en lípidos y calorías totales y preeclampsia materna $(13,20)$.

\section{CLASIFICACIÓN}

La clasificación internacional actual de la retinopatía del prematuro describe la localización, extensión y gravedad de la enfermedad $(1,3)$.

\section{Estadios (4)}

- Estadio 1. Línea de demarcación: Una línea fina, blanca que separa la retina vascular de la avascular.

- Estadio 2. Cresta monticular: La línea de demarcación que aparece en el estadio 1 aumenta de volumen y se extiende fuera del plano de la retina.

- Estadio 3. Existe un crecimiento de tejido vascular hacia el espacio vítreo.

- Estadio 4. Desprendimiento de retina parcial. Se subdivide en $A$, si no involucra mácula y en $B$, si involucra (mácula desprendida).

- Estadio 5. Desprendimiento de retina total. 


\section{FIGURA 1. Clasificación internacional de la retinopatía del prematuro por zonas}

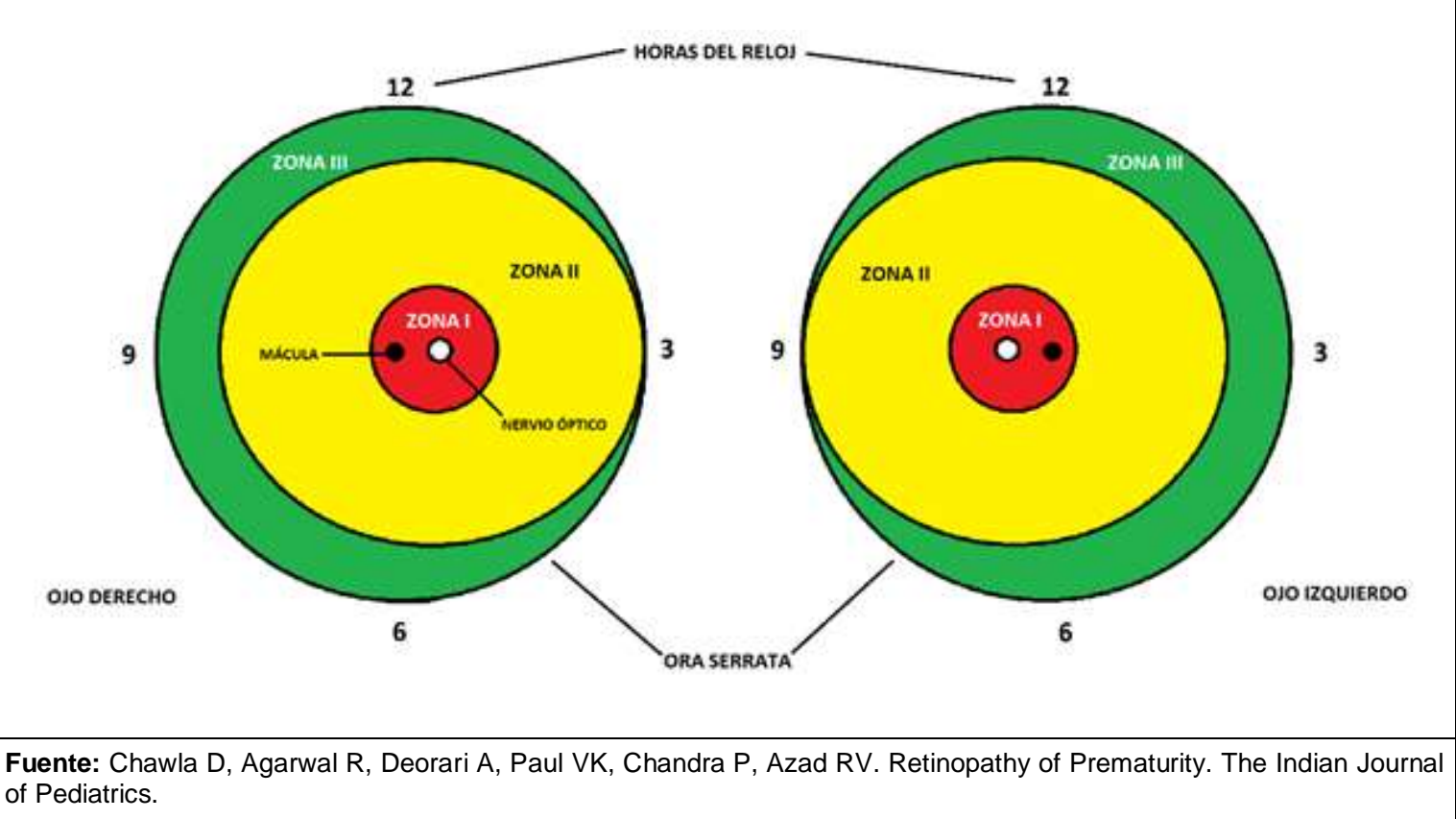

La enfermedad Plus, es un término descriptivo que se refiere a la dilatación y tortuosidad de los vasos del polo posterior e indica que hay actividad. Puede acompañar a cualquier estadio de retinopatía. La enfermedad Pre Plus, son vasos más tortuosos y dilatados de lo esperado pero sin llegar a las características de enfermedad Plus $(4,22,23)$.

\section{Localización (3)}

Zona I (polo posterior): se extiende hasta el doble de la distancia entre la papila y la mácula, $30^{\circ}$ alrededor del nervio óptico.

Zona II (intermedia): ocupa desde el límite externo de la zona I hasta la ora serrata en el lado nasal y ecuador anatómico temporal.

Zona III (externa): Semiluna restante que abarca desde el borde externo de la zona II hasta la ora serrata en el lado temporal.

\section{Extensión}

Se describe la extensión de la retinopatía por sectores horarios (4). Ver FIGURA 1

- Retinopatía umbral: Es una retinopatía en estadio 3 con presencia de enfermedad Plus en zona I o II y que se extiende al menos en 5 horas continuas $u$ horas acumulativas.

- Retinopatía pre umbral: Definida como cualquier retinopatía en zona I que sea menos que la umbral, 0 en zona II con estadio 2 y enfermedad plus, o zona II con estadio 3 sin enfermedad plus 0 zona II con estadio 3 con enfermedad plus pero menos de 5 horas continuas u 8 horas acumulativas.

- Retinopatía agresiva posterior: Es una forma grave y rápidamente progresiva, de localización posterior y con enfermedad Plus. Más comúnmente en zona I. 
A mayor estadio o menor zona, es peor la ROP (1).

\section{CLÍNICA}

En más del $90 \%$ de los niños con riesgo la evolución conduce a una resolución y regresión espontánea, sin apenas efectos residuales ni alteración visual. Menos del $10 \%$ de los niños progresan hacia una enfermedad grave, con vasoproliferación extraretiniana significativa, cicatrización, desprendimiento de retina y deterior de visión. Algunos niños con retinopatía del prematuro abortiva 0 involucionada conservan la línea de demarcación, con vascularización deficiente en la retina periférica 0 ramificación anormal, tortuosidad 0 rectificación de los vasos retinianos. En ocasiones quedan alteraciones pigmentarias retinianas, tracción sobre la retina (denominada "papila arrastrada"), ectopia macular, pliegues o roturas retinianas. En otros se produce un desprendimiento de retina total, que a menudo adopta una configuración en embudo.

El cuadro clínico se caracteriza entonces por una membrana retrolental que origina leucocoria. Algunos pacientes desarrollan catarata, glaucoma y signos inflamatorios. El estadio final es el de un ojo ciego y doloroso o una ptisis degenerativa. Hasta el $80 \%$ de los niños con historia de ROP severa desarrollaran estrabismo durante los primeros 6 años de vida.

El espectro en la retinopatía del prematuro también incluye miopía, a menudo progresiva y de magnitud considerable, en lactantes. También aumenta la anisometropía, estrabismo, ambliopía y nistagmo (3).

\section{DIAGNÓSTICO Y TAMIZAJE}

Las recomendaciones según la American Academy of Pediatrics indican realizar un examen de fondo de ojo con dilatación como tamizaje en infantes con peso $<1500 \mathrm{~g} 0$ de 30 semanas o menos. Así como en infantes con una edad gestacional mayor pero con evolución clínica inestable incluyendo los que precisen soporte cardiorrespiratorio y aquellos casos considerados de riesgo por el neonatólogo encargado $(25,22,26,6,3)$. En Costa Rica según la encuesta de expertos para Latinoamérica y el Caribe del 2015, los criterios de tamizaje para ROP se realizan en infantes con peso $<1500 \mathrm{~g}$ y edad gestacional $<34$ semanas. En dicha encuesta de los 706 neonatos tamizados únicamente 22 requirieron tratamiento (7).

\section{MÉTODO DE CRIBADO}

La primera exploración del fondo de ojo para valorar la presencia de retinopatía se realiza a la cuarta semana de vida si la edad gestacional fue $>28$ semanas $y$ a la quinta semana de vida si la edad gestacional fue $<28$ semanas. Los controles sucesivos de realizan cada 2 a 3 semanas hasta que la retina complete su vascularización. Los niños que desarrollan una retinopatía se controlan cada semana hasta que se observan signos de regresión o hasta que la retinopatía progresa a un estadio que requiera tratamiento $(4,6)$.

El método para realizar el examen de fondo de ojo con dilatación se recomienda realizarse con oftalmoscopio binocular indirecto tras dilatar las pupilas con Cyclomydril $(0.2 \%$ ciclopentolato y $1.0 \%$ fenilefrina $)$ $(1,4)$. 
TABLA 3. Clasificación de la la ROP segun el tratamiento temprano

\begin{tabular}{|l|l|}
\hline \multicolumn{1}{|c|}{ ROP tipo 1 } & \multicolumn{1}{c|}{ ROP tipo 2} \\
\hline Zona I, cualquier estadio con enfermedad plus & Zona I, estadio 1 o 2 sin enfermedad plus \\
\hline Zona I, estadio 3 sin enfermedad plus & Zona II, estadio 3 sin enfermedad plus \\
\hline Zona II, estadio 2 o 3 con enfermedad plus & \\
\hline $\begin{array}{l}\text { Fuente: Lueder G, Archer, MD, S, Hered, MD, R, Karr D, Kodsi S. 2018-2019 Basic and Clinical Science Course, } \\
\text { Section 06: Pediatric Ophthalmology and Strabismus. American Academy of Ophthalmology }\end{array}$ \\
\hline
\end{tabular}

\section{TRATAMIENTO Y PREVENCIÓN}

Profilácticamente para la retinopatía del prematuro consiste en disminuir los factores de riesgo como la ventilación artificial a largo plazo y la suplementación con oxígeno no controlada (23).

Inicialmente la crioterapia emergió en 1980 como la terapia estándar para ROP, que luego fue sustituida con la ablación láser que actualmente se considera como el "gold standard" $(6,15)$. Para determinar el tratamiento la American Academy of Opthalmology recomienda utilizar el estudio Early Treatment for Retinopathy of Prematurity (ETROP), el cual utiliza la clasificación de riesgo (1). Ver TABLA 3.

El Grupo colaborador para el tratamiento temprano de la ROP ETROP (Early Treatment ROP) concluyó que el tratamiento temprano en pacientes con clasificación tipo I o en estadio pre umbral, ha demostrado mejores resultados estructurales y anatómicos que el tratamiento convencional. Terapia láser está altamente recomendada para cualquier ojo con tipo I, aunque negativamente se ha visto que los infantes tratados con láser transpupilar tienen un riesgo aumentado de miopía de hasta un $70 \%$. Pacientes con tipo 2 deben ser observados de cerca y seguimiento periódico para evitar progresión a enfermedad tipo I $(1,3,6,24)$.

Actualmente se están llevando a cabo ensayos clínicos que utilizan propranolol e inyecciones intra vítreas de antagonistas del VEGF para evaluar su eficacia y riesgo. El propranolol con el fin de reducir la neovascularización retiniana $(3,6)$. En el estudio The Bevacizumab Eliminates the Angiogenic Threat of Retinopathy of Prematurity (BEAT-ROP) evaluó la eficacia del Bevacizumab intravítreo como monoterapia para el tratamiento de ROP. El estudio mostró los beneficios significativos de Bevacizumab versus terapia con láser en el tratamiento de la ROP zona 1, etapa 3+. La terapia fotocoagulación con láser puede causar la destrucción en la vasculatura retiniana periférica que conduce a la pérdida permanente del campo de la visión periférica. Por el contrario, el Bevacizumab intravítreo permite el crecimiento normal continuo del vaso en la retina periférica, preservando así una mejor visión periférica. Actualmente el Bevacizumab no está aprobado por la FDA para el tratamiento de la ROP $(15,27)$.

\section{PREVENCIÓN}

En Costa Rica existe una política gubernamental que incluye prevención, detección, tratamiento del ROP mediante el tamizaje, el cual es 
financiado por un programa de seguridad social. Este protocolo fue creado por oftalmólogos y neonatólogos reconocidos por el ministerio de salud. En esta legislación es mandatorio realizar un fondo de ojo en infantes pretérminos (7).

La prevención de la ROP depende sobretodo de la prevención de partos prematuros y sus problemas asociados. Sin embargo, se ha estudiado la implicación de otros posibles factores con el objeto de reducir la aparición de ROP entre los prematuros. Algunos estudios han considerado que la luz ambiental podría ser un posible agente inductor, pero el estudio LIGHT-ROP estableció rotundamente que la reducción de la luz ambiental no tenía impacto alguno sobre la ROP. Con respecto a la asociación entre ROP y saturación de oxígeno, las investigaciones recientes se han enfocado en mantener a los lactantes muy prematuros con saturaciones de oxígeno lo suficientemente bajas como para reducir el riesgo de ROP y lo suficientemente altas como para optimizar la supervivencia (3).

\section{CONCLUSIÓN}

La ROP es una enfermedad de la retina potencialmente devastadora que se desarrolla en bebés prematuros de bajo peso al nacer. El desarrollo de un estándar internacional para el tamizaje, el diagnóstico y el tratamiento de la ROP ha sido esencial para mejorar los resultados. La atención neonatal ha mejorado en el tiempo, por lo que ha venido en aumento la incidencia de ROP debido a una mayor supervivencia en niños prematuros. El avance más reciente es el uso exitoso "off-label" de agentes anti-VEGF intraoculares para enfermedad grave. Sin embargo, para la gran mayoría de ROP, el tratamiento con láser es altamente efectivo y predecible. Para prevenir las causas tratables de pérdida de la visión, el seguimiento adecuado es muy importante para todos los bebés prematuros con o sin antecedentes de ROP. Este seguimiento en los pacientes con ROP impactará de manera significativa en términos de salud en general, salud visual, capacidad, personalidad e impacto familiar.

\section{REFERENCIAS}

1. Lueder G, Archer, MD, S, Hered, MD, R, Karr D, Kodsi S. 2018-2019 BCSC (Basic and Clinical Science Course), Section 06: Pediatric Ophthalmology and Strabismus (MAJOR REVISION). 2017. San Francisco, CA: American Academy of Ophthalmology. Disponible en: https://www.aao.org/assets/5e0f04a7-77a1-457b-81af2f650333faae/636312517616000000/bcsc1718-s06-pdf

2. Messa AA, Mattos RB, Areco KCN, Sallum JMF. Vision-related quality of life in children with retinopathy of prematurity. Arquivos Brasileiros de Oftalmologia. 2015;78(4):224-228. https://doi.org/10.5935/0004-2749.20150058

3. Kliegman R, Stanton B, Geme J, Schor N. Nelson. Tratado de pediatría. 20ed.. Elsevier; 201606. Disponible en: https://tienda.elsevierhealth.com/nelson-tratado-de-pediatria-9788491130178.html

4. Camba Longueira F, Perapoch López J, Martín Begué N. Retinopatía de la prematuridad. Protocolos Diagnóstico Terapeúticos de la AEP: Neonatología. 2008. Disponible en: https://www.aeped.es/sites/default/files/documentos/46.pdf 
5. Asano MK, Dray PB. Retinopathy of prematurity. Disease-a-Month. 2014 06;60(6):282-291. https://doi.org/10.1016/j.disamonth.2014.03.009

6. Hellström A, Smith LE, Dammann O. Retinopathy of prematurity. The Lancet. 2013 Oct;382(9902):1445-1457. https://doi.org/10.1016/s0140-6736(13)60178-6

7. Arnesen L, Durán P, Silva J, Brumana L. A multi-country, cross-sectional observational study of retinopathy of prematurity in Latin America and the Caribbean. Rev Panam Salud Publica. 2016;39(6):322-29. Disponible en: http://iris.paho.org/xmlui/handle/123456789/28539

8. Gunn DJ, Cartwright DW, Gole GA. Prevalence and outcomes of laser treatment of aggressive posterior retinopathy of prematurity. Clinical \& Experimental Ophthalmology. 201401 23;42(5):459465. https://doi.org/10.1111/ceo.12280

9. Hartnett ME. Advances in understanding and management of retinopathy of prematurity. Survey of Ophthalmology. 2017 05;62(3):257-276. https://doi.org/10.1016/j.survophthal.2016.12.004

10. Smith LE. Pathogenesis of retinopathy of prematurity. Seminars in Neonatology. 2003 Dec;8(6):469473. https://doi.org/10.1016/s1084-2756(03)00119-2

11. Cayabyab R, Ramanathan R. Retinopathy of Prematurity: Therapeutic Strategies Based on Pathophysiology. Neonatology. 201606 03;109(4):369-376. https://doi.org/10.1159/000444901

12. Gilbert C. Retinopathy of prematurity: A global perspective of the epidemics, population of babies at risk and implications for control. Early Human Development. 2008 02;84(2):77-82. https://doi.org/10.1016/i.earlhumdev.2007.11.009

13. Jordan CO. Retinopathy of Prematurity. Pediatric Clinics of North America. 2014 06;61(3):567-577. https://doi.org/10.1016/j.pcl.2014.03.003

14. Good W, Hardy R, Dobson V, Palmer E, Phelps D, Quintos M, Tung B. The Incidence and Course of Retinopathy of Prematurity: Findings From the Early Treatment for Retinopathy of Prematurity Study. PEDIATRICS. 200507 01;116(1):15-23. https://doi.org/10.1542/peds.2004-1413

15. Quimson SK. Retinopathy of Prematurity: Pathogenesis and Current Treatment Options. Neonatal Network. 2015;34(5):284-287. https://doi.org/10.1891/0730-0832.34.5.284

16. Isaza G, Donaldson L, Chaudhary V. Increased incidence of retinopathy of prematurity and evolving treatment modalities at a Canadian tertiary centre. Canadian Journal of Ophthalmology. 2018 07;. https://doi.org/10.1016/i.jcjo.2018.05.005

17. Hartnett ME, Lane RH. Effects of oxygen on the development and severity of retinopathy of prematurity. Journal of American Association for Pediatric Ophthalmology and Strabismus. 2013 06;17(3):229-234. https://doi.org/10.1016/j.jaapos.2012.12.155

18. Saugstad OD. Oxygen and retinopathy of prematurity. Journal of Perinatology. 200604 25;26(S1):S46-S50. https://doi.org/10.1038/sj.jp.7211475

19. Ortega-Molina JM, Anaya-Alaminos R, Uberos-Fernández J, Solans-Pérez de Larraya A, ChavesSamaniego MJ, Salgado-Miranda A, Piñar-Molina R, Jerez-Calero A, García-Serrano JL. Genetic and Environmental Influences on Retinopathy of Prematurity. Mediators of Inflammation. 2015;2015:1-7. https://doi.org/10.1155/2015/764159

20. Dai AI, Demiryürek S, Aksoy SN, Perk P, Saygili O, Güngör K. Maternal Iron Deficiency Anemia as a Risk Factor for the Development of Retinopathy of Prematurity. Pediatric Neurology. 2015 08;53(2):146-150. https://doi.org/10.1016/j.pediatrneurol.2015.04.002 
21. Zhou J, Shukla VV, John D, Chen C. Human Milk Feeding as a Protective Factor for Retinopathy of Prematurity: A Meta-analysis. PEDIATRICS. 2015 Nov 16;136(6):e1576-e1586. https://doi.org/10.1542/peds.2015-2372

22. Chawla D, Agarwal R, Deorari A, Paul VK, Chandra P, Azad RV. Retinopathy of Prematurity. The Indian Journal of Pediatrics. 2010 Oct 27;79(4):501-509. https://doi.org/10.1007/s12098-010-0279-7

23. Stahl A, Göpel W. Screening and Treatment in Retinopathy of Prematurity. Deutsches Aerzteblatt Online. 2015 Oct 23;. https://doi.org/10.3238/arztebl.2015.0730

24. Good WV, Early Treatment for Retinopathy of Prematurity Cooperative Group. Final results of the Early Treatment for Retinopathy of Prematurity (ETROP) randomized trial. Trans Am Ophthalmol Soc. 2004;102:233-48; discussion 248-50. Disponible en: https://www.ncbi.nlm.nih.gov/pmc/articles/PMC1280104/

25. Casteels I, Cassiman C, Van Calster J, Allegaert K. Educational paper. European Journal of Pediatrics. 2011 Nov 04;171(6):887-893. https://doi.org/10.1007/s00431-011-1610-7

26. Fierson W, Saunders R, Good W, Palmer E, Phelps D, Reynolds J, Chiang M. Screening Examination of Premature Infants for Retinopathy of Prematurity. PEDIATRICS. 2012 Dec 31;131(1):189-195. https://doi.org/10.1542/peds.2012-2996

27. Sternberg P, Durrani AK. Evolving Concepts in the Management of Retinopathy of Prematurity. American Journal of Ophthalmology. 2018 02;186:xxiii-xxxii. https://doi.org/10.1016/j.ajo.2017.10.027 